\title{
Optical signatures of non-Markovian behavior in open quantum systems
}

\section{McCutcheon, Dara}

\section{Published in:}

Physical Review A

Link to article, DOI:

10.1103/PhysRevA.93.022119

Publication date:

2016

\section{Document Version}

Publisher's PDF, also known as Version of record

Link back to DTU Orbit

Citation (APA):

McCutcheon, D. (2016). Optical signatures of non-Markovian behavior in open quantum systems. Physical Review A, 93(2), [022119]. https://doi.org/10.1103/PhysRevA.93.022119

\section{General rights}

Copyright and moral rights for the publications made accessible in the public portal are retained by the authors and/or other copyright owners and it is a condition of accessing publications that users recognise and abide by the legal requirements associated with these rights.

- Users may download and print one copy of any publication from the public portal for the purpose of private study or research.

- You may not further distribute the material or use it for any profit-making activity or commercial gain

- You may freely distribute the URL identifying the publication in the public portal

If you believe that this document breaches copyright please contact us providing details, and we will remove access to the work immediately and investigate your claim. 


\title{
Optical signatures of non-Markovian behavior in open quantum systems
}

\author{
Dara P. S. McCutcheon \\ Department of Photonics Engineering, DTU Fotonik, Ørsteds Plads, 2800 Kongens Lyngby, Denmark
}

(Received 30 April 2015; revised manuscript received 4 December 2015; published 25 February 2016)

\begin{abstract}
We derive an extension to the quantum regression theorem which facilitates the calculation of two-time correlation functions and emission spectra for systems undergoing non-Markovian evolution. The derivation exploits projection operator techniques, with which we obtain explicit equations of motion for the correlation functions, making only a second-order expansion in the system-environment coupling strength and invoking the Born approximation at a fixed initial time. The results are used to investigate a driven semiconductor quantum dot coupled to an acoustic phonon bath, where we find the non-Markovian nature of the dynamics has observable signatures in the form of phonon sidebands in the resonance fluorescence emission spectrum. Furthermore, we use recently developed non-Markovianity measures to demonstrate an associated flow of information from the phonon bath back into the quantum dot exciton system.
\end{abstract}

DOI: 10.1103/PhysRevA.93.022119

\section{INTRODUCTION}

Two-time correlation functions are quantities of frequent interest in many areas of physics. This is particularly true in quantum optics, where correlation functions of the form $\left\langle A\left(t_{1}\right) B\left(t_{2}\right)\right\rangle$ give the field correlation properties of an emitting system such as a driven atom, and whose Fourier transform gives the measured spectrum [1]. If the governing Hamiltonian can be diagonalized exactly, calculation of the two-time correlation function is no more challenging than calculating a one-time expectation value of the form $\left\langle A\left(t_{1}\right)\right\rangle$. However, it is more often the case that the emitting system is an open system, whose dynamics can only be approximated. In this case, since the system operators $A$ and $B$ are evaluated at two distinct times, calculation of the correlation function given knowledge of system dynamics alone is not at first sight straightforward. The quantum regression theorem, however, gives a prescription of how such correlation functions can be related to more readily obtainable system expectation values [2]. A subtle caveat of the quantum regression theorem, however, is that it applies only to systems undergoing strictly Markovian evolution. It requires that the complete density operator of the system and environment factorizes at all times, and that the reduced system density operator obeys a time-independent master equation [3-10].

The requirement of Markovian evolution is typically fulfilled in the traditional case of atomic quantum optics due to the extremely short correlation time of the electromagnetic environment [11,12]. However, more recent technological advances in the fabrication of artificial emitters and the engineering of structured environments have given rise to systems whose evolution is not purely Markovian, yet whose properties are typically probed optically. These systems include semiconductor quantum dots (QDs), for which Rabi oscillations [13-15], resonance fluorescence [16-20], and single-photon emission [21-23] have all been demonstrated. QDs, however, exist in a solid-state substrate, and interactions with phonons and nuclear spins can modify their emission properties [15,24-26] and also give rise to non-Markovian behavior [27-31]. Additionally, for technological applications, such as indistinguishable and entangled photon sources [32-35], it is often desirable to place artificial emitters in structured photonic environments such as in photonic crystals or micropillar cavities, which also have the potential to lead to non-Markovian behavior.

Thus, in order to model the optical properties of these ever more exotic systems, it is important to establish how two-time correlation functions can be calculated for open systems undergoing non-Markovian evolution. We note that efforts in this direction have been made [3-10], and the conditions under which the regression theorem holds have been scrutinized [7]. Many of these, however, rely on a number of uncontrolled approximations, such as artificially enforcing time locality [8,9] or assuming a restrictive (rotating-wave-like) form of the system-environment coupling [4,5]. Additionally, it is not clear to what extent non-Markovian behavior has any measurable optical consequences in physically relevant systems.

In this work we use projection operator techniques to derive a non-Markovian extension to the quantum regression theorem, valid to second order in the system-environment coupling strength, and invoking the Born approximation only at a single fixed initial time. The second-order expansion restricts the theory to weak-system environment coupling regimes for which non-Markovian behavior is typically only present for short times and which is usually very challenging to observe. The key advantage of the present work, however, is that this short-time behavior is of a two-time correlation function, whose spectral counterpart corresponds to a concrete readily measurable quantity. Specifically, we apply our formalism to the relevant case of a driven QD [16-20] and find that the experimentally observed phonon sidebands in the emission spectra are a direct consequence of non-Markovian behavior, which the standard Markovian treatment fails to capture. Moreover, we confirm true non-Markovianity and indivisibility of the underlying dynamical map by demonstrating that the phonon sidebands are associated with a flow of information from the phonon environment back into the QD system [36].

\section{TWO-TIME CORRELATION FUNCTIONS AND THE REGRESSION THEOREM}

We begin by introducing two-time correlation functions and the standard (Markovian) regression theorem. We consider a system $S$ interacting with an environment $E$, and 
wish to calculate two-time correlation functions of the form $G(t, \tau)=\langle A(t+\tau) B(t)\rangle=\operatorname{Tr}_{S+E}[A(t+\tau) B(t) \chi(0)]$, where $A$ and $B$ are system operators, $\chi(0)$ is the total system-plusenvironment state at $t=0$, and $\operatorname{Tr}_{S+E}$ denotes a trace over both $S$ and $E$. For a time-independent Hamiltonian $H$ we have $A(t)=U^{\dagger}(t) A U(t)$ with $U(t)=\exp [-i H t]$ (we set $\left.\hbar=1\right)$, and using the cyclic property of the trace we find

$$
G(t, \tau)=\operatorname{Tr}_{S}[A \Lambda(t, \tau)],
$$

where the system operator $\Lambda(t, \tau)$ is given by

$$
\Lambda(t, \tau)=\operatorname{Tr}_{E}\left[U(\tau) B \chi(t) U^{\dagger}(\tau)\right]
$$

with $\chi(t)=U(t) \chi(0) U^{\dagger}(t)$. From Eq. (1) we see that calculation of $G(t, \tau)$ amounts to calculating something analogous to the expectation value of $A$, but with respect to the operator $\Lambda(t, \tau)$ rather than the reduced system density operator $\rho(t)=$ $\operatorname{Tr}_{E}\left[U(t) \chi(0) U^{\dagger}(t)\right]$. For this reason we refer to $\Lambda(t, \tau)$ as the reduced effective density operator and $\rho(t)$ as the reduced physical density operator.

The standard regression theorem proceeds by observing that the definition of the effective density operator $\Lambda(t, \tau)$ in Eq. (2) bears a strong resemblance to that of the reduced physical density operator, $\rho(t)=\operatorname{Tr}_{E}\left[U(t) \chi(0) U^{\dagger}(t)\right]$. As such, if we know the equation of motion for the physical density operator with respect to $t$, say $\partial_{t} \rho(t)=\Phi \rho(t)$, then the reduced effective density operator will obey the same equation of motion but with respect to $\tau$, and with a modified initial condition, namely $\partial_{\tau} \Lambda(t, \tau)=\Phi \Lambda(t, \tau)$ and $\Lambda(t, 0)=B \rho(t)$. We will see, however, that this procedure contains a hidden assumption that the total physical density operator $\chi(t)$ factorizes for all times [3-5,8].

\section{A. Effective density operator master equation using projection operators}

To see how this assumption arises, and how it can be removed, we now derive the quantum regression theorem using the projection operator formalism [37-40]. This wellestablished formalism was originally developed to calculate physical density operator master equations, and our purpose here is to do the same for the effective density operator, taking particular care to identify places where any approximations have different physical significance. To begin we must establish an interaction picture for the total effective density operator, which we define as $\Upsilon(t, \tau)=U(\tau) B \chi(t) U^{\dagger}(\tau)$, such that $\Lambda(t, \tau)=\operatorname{Tr}_{E}[\Upsilon(t, \tau)]$. We write the total Hamiltonian $H=H_{0}+\alpha H_{I}$, where $H_{0}=H_{S}+H_{E}$ with $H_{S}$ and $H_{E}$ acting exclusively on $S$ and $E$ respectively. We recall that the unitary operators $U(\tau)$ and $U_{0}(\tau)$ are defined as the solutions to the differential equations $i \partial_{\tau} U(\tau)=H U(\tau)$ and $i \partial_{\tau} U_{0}(\tau)=H_{0} U_{0}(\tau)$, and the interaction picture effective density operator as $\tilde{\Upsilon}(t, \tau)=U_{I}(\tau) B \chi(t) U_{I}^{\dagger}(\tau)$ with $U_{I}(\tau)=$ $U_{0}^{\dagger}(\tau) U(\tau)$. From these definitions we find

$$
\partial_{\tau} \tilde{\Upsilon}(t, \tau)=-i \alpha\left[\tilde{H}_{I}(\tau), \tilde{\Upsilon}(t, \tau)\right]=\alpha \mathcal{L}(\tau) \tilde{\Upsilon}(t, \tau),
$$

where $\tilde{H}_{I}(\tau)=U_{0}^{\dagger}(\tau) H_{I} U_{0}(\tau)$ and the Liouvillian $\mathcal{L}(\tau)$ is defined to satisfy the second equality. We naturally define $\tilde{\Lambda}(t, \tau)=\operatorname{Tr}_{E}[\tilde{\Upsilon}(t, \tau)]$, and note that since we can write $U_{0}(\tau)=U_{S}(\tau) U_{E}(\tau)$ with the subscripts indicating whether the operators act on $S$ or $E$ we find $\Lambda(t, \tau)=$ $U_{S}(\tau) \tilde{\Lambda}(t, \tau) U_{S}^{\dagger}(\tau)$. The Schrödinger and interaction picture equations of motion are then related through

$$
\partial_{\tau} \Lambda(t, \tau)=i\left[\Lambda(t, \tau), H_{S}\right]+U_{S}(\tau)\left[\partial_{\tau} \tilde{\Lambda}(t, \tau)\right] U_{S}^{\dagger}(\tau) .
$$

These results demonstrate that the effective density operator has a well-defined interaction picture which facilitates the use of the master equation techniques below.

We now introduce the projection operators $\mathcal{P}$ and $\mathcal{Q}=$ $(\mathbb{1}-\mathcal{P})$, which are defined through [38-40]

$$
\mathcal{P} \tilde{\Upsilon}(t, \tau)=\operatorname{Tr}_{E}[\tilde{\Upsilon}(t, \tau)] \otimes \rho_{R}=\tilde{\Lambda}(t, \tau) \otimes \rho_{R},
$$

where $\rho_{R}$ is a reference state of the environment. The projection operators project the effective density operator into factorizing and nonfactorizing components; i.e., we can write $\tilde{\Upsilon}(t, \tau)=(\mathcal{P}+\mathcal{Q}) \tilde{\Upsilon}(t, \tau)$, where the first term factorizes by definition and the second term captures those components which do not. From these basic definitions one can show that $\mathcal{P}^{2}=\mathcal{P}$ and $\mathcal{Q}^{2}=\mathcal{Q}$, while $\mathcal{Q P}=\mathcal{P} \mathcal{Q}=0$. In what follows we assume $\operatorname{Tr}_{E}\left[H_{I} \rho_{R}\right]=0$. This is not an approximation, since if $\operatorname{Tr}_{E}\left[H_{I} \rho_{R}\right]=\left\langle H_{I}\right\rangle \neq 0$ we can redefine $H_{S}^{\prime}=H_{S}+\left\langle H_{I}\right\rangle$ and $H_{I}^{\prime}=H_{I}-\left\langle H_{I}\right\rangle$, leaving the total Hamiltonian unchanged, and we then have $\operatorname{Tr}_{E}\left[H_{I}^{\prime} \rho_{E}\right]=$ 0 by definition $[41,42]$. Provided our reference state is chosen such that $\left[H_{E}, \rho_{E}\right]=0$, valid for, e.g., thermal states, we find $\operatorname{Tr}_{E}\left[\tilde{H}_{I}^{\prime}(\tau) \rho_{R}\right]=0$, which implies $\mathcal{P} \mathcal{L}(\tau) \mathcal{P}=0$.

Now, our aim is to derive an equation of motion for the factorizing part of the effective density operator $\mathcal{P} \tilde{\Upsilon}(t, \tau)$, from which we can readily obtain $\Lambda(t, \tau)=\operatorname{Tr}_{E}[\mathcal{P} \tilde{\Upsilon}(t, \tau)]$, and using Eq. (1) calculate the two-time correlation function. Following Ref. [37] we act with both $\mathcal{P}$ and $\mathcal{Q}$ on Eq. (3), yielding two differential equations which we must solve simultaneously. By inserting $\mathbb{1}=\mathcal{P}+\mathcal{Q}$ on the right-hand side and using $\mathcal{P} \mathcal{L}(\tau) \mathcal{P}=0$ the first of these becomes

$$
\partial_{\tau} \mathcal{P} \tilde{\Upsilon}(t, \tau)=\alpha \mathcal{P} \mathcal{L}(\tau) \mathcal{Q} \tilde{\Upsilon}(t, \tau)
$$

while the second involving $\partial_{\tau} \mathcal{Q} \tilde{\Upsilon}(t, \tau)$ can be formally integrated to give

$$
\begin{aligned}
\mathcal{Q} \tilde{\Upsilon}(t, \tau)= & G_{F}(\tau, 0) \mathcal{Q} \tilde{\Upsilon}(t, 0) \\
& +\alpha \int_{0}^{\tau} d s G_{F}(\tau, s) \mathcal{Q L}(s) \mathcal{P} \tilde{\Upsilon}(t, s),
\end{aligned}
$$

where $G_{F}(\tau, s)=\mathrm{T}_{\leftarrow} \exp \left[\alpha \int_{s}^{\tau} \mathrm{d} s^{\prime} \mathcal{Q} \mathcal{L}\left(s^{\prime}\right)\right]$ with $\mathrm{T}_{\leftarrow}$ being the chronological time ordering operator [37]. To obtain a timelocal form, from Eq. (3) we see that we can write $\tilde{\Upsilon}(t, s)=$ $G_{B}(\tau, s) \tilde{\Upsilon}(t, \tau)$, where $G_{B}(\tau, s)=\mathrm{T}_{\rightarrow} \exp \left[-\alpha \int_{s}^{\tau} d s^{\prime} \mathcal{L}\left(s^{\prime}\right)\right]$ with $\mathrm{T}_{\rightarrow}$ being the antichronological time ordering operator. From Eq. (7) we then find

$$
[\mathbb{1}-\Sigma(\tau)] \mathcal{Q} \tilde{\Upsilon}(t, \tau)=G_{F}(\tau, 0) \mathcal{Q} \tilde{\Upsilon}(t, 0)+\Sigma(\tau) \mathcal{P} \tilde{\Upsilon}(t, \tau)
$$

where $\Sigma(\tau)=\alpha \int_{0}^{\tau} d s G_{F}(\tau, s) \mathcal{Q L}(s) \mathcal{P} G_{B}(\tau, s)$. Provided the inverse of the operator $[\mathbb{1}-\Sigma(\tau)]$ exists, Eq. (8) can be solved for $\mathcal{Q} \tilde{\Upsilon}(t, \tau)$. Since we are ultimately interested in the weakcoupling limit of the system-environment interaction $\alpha$, and since $\Sigma(\tau)$ contains no zeroth-order term in $\alpha$, we assume the existence of such an operator, and in solving for $\mathcal{Q} \tilde{\Upsilon}(t, \tau)$ 
we obtain

$$
\begin{aligned}
\mathcal{Q} \tilde{\Upsilon}(t, \tau)= & {[\mathbb{1}-\Sigma(\tau)]^{-1} \Sigma(\tau) \mathcal{P} \tilde{\Upsilon}(t, \tau) } \\
& +[\mathbb{1}-\Sigma(\tau)]^{-1} G_{F}(\tau, 0) \mathcal{Q} \tilde{\Upsilon}(t, 0) .
\end{aligned}
$$

Inserting this formal solution for the nonfactorizing component of the effective density operator into Eq. (6) for the factorising component we find

$$
\partial_{\tau} \mathcal{P} \tilde{\Upsilon}(t, \tau)=\mathcal{I}(\tau) \mathcal{Q} \tilde{\Upsilon}(t, 0)+\mathcal{K}(\tau) \mathcal{P} \tilde{\Upsilon}(t, \tau),
$$

where we have defined the kernels

$$
\begin{gathered}
\mathcal{I}(\tau)=\alpha \mathcal{P} \mathcal{L}(\tau)[\mathbb{1}-\Sigma(\tau)]^{-1} G_{F}(\tau, 0) \mathcal{Q}, \\
\mathcal{K}(\tau)=\alpha \mathcal{P} \mathcal{L}(\tau)[\mathbb{1}-\Sigma(\tau)]^{-1} \Sigma(\tau) \mathcal{P}
\end{gathered}
$$

These expressions constitute an exact equation of motion for the reduced effective density operator, with an inhomogeneous term which depends on the physical density operator through $\mathcal{Q} \tilde{\Upsilon}(t, 0)=\mathcal{Q} B \chi(t)$.

For these reasons, in what follows it will be useful to also consider the evolution for the factorizing and nonfactorizing parts of the physical density operator $\chi(t)$. For this purpose we use the projection operator methods outlined above, and we find that the derivation proceeds in precisely the same manner, with the only difference being that the time argument $\tau$ is replaced with $t$ and the initial condition is $(\mathcal{P}+\mathcal{Q}) \chi(0)$. In exact analogy with Eq. (9), we find that the nonfactorizing part has solution

$$
\begin{aligned}
\mathcal{Q} \tilde{\chi}(t)= & {[\mathbb{1}-\Sigma(t)]^{-1} \Sigma(t) \mathcal{P} \tilde{\chi}(t) } \\
& +[\mathbb{1}-\Sigma(t)]^{-1} G_{F}(t, 0) \mathcal{Q} \tilde{\chi}(0),
\end{aligned}
$$

leading to the equation of motion

$$
\partial_{t} \mathcal{P} \tilde{\chi}(t)=\mathcal{I}(t) \mathcal{Q} \tilde{\chi}(0)+\mathcal{K}(t) \mathcal{P} \tilde{\chi}(t),
$$

with the kernels again given by Eqs. (11) and (12).

\section{B. Removal of the Born approximation and the non-Markovian regression theorem}

Returning to Eq. (10) for the effective density operator, we now consider the inhomogeneous term $\mathcal{I}(\tau) \mathcal{Q} \tilde{\Upsilon}(t, 0)$. If we were to make the Born approximation, and assume that the physical density operator factorizes at all times, $\chi(t) \approx \rho(t) \otimes$ $\rho_{R}$, then $\mathcal{Q} \tilde{\Upsilon}(t, 0)=0$ and the inhomogeneous term vanishes. Analogously, in Eq. (14) we see that in assuming factorizing initial conditions, $\chi(0) \approx \rho(0) \otimes \rho_{R}$, the inhomogeneous term for the physical density operator vanishes. In these cases the equations of motion for the effective and the physical density operator become identical; i.e., we have $\partial_{t} \mathcal{P} \tilde{\chi}(t)=\mathcal{K}(t) \mathcal{P} \tilde{\chi}(t)$ and $\partial_{\tau} \mathcal{P} \tilde{\Upsilon}(t, \tau)=\mathcal{K}(\tau) \mathcal{P} \tilde{\Upsilon}(t, \tau)$. We conclude that we must make the Born approximation at all times for the standard regression theorem to apply.

We now turn to the key insight of this work, which allows us to remove the Born approximation. Since $B$ is a system operator, and assuming $\left[H_{E}, \rho_{R}\right]=0$, it can be shown that $\mathcal{Q} \tilde{\Upsilon}(t, 0)=B \mathcal{U}(t) \mathcal{Q} \tilde{\chi}(t)$, where $\mathcal{U}_{0}(t) \tilde{\chi}(t)=U_{0}(t) \tilde{\chi}(t) U_{0}^{\dagger}(t)$. The object $\mathcal{Q} \tilde{\chi}(t)$ represents deviations from factorability of the physical density operator. However, we already have an exact form for this, namely Eq. (13). Assuming factorizing initial conditions only, the second term in Eq. (13) is zero, and using what remains in Eq. (10) gives

$$
\partial_{\tau} \mathcal{P} \tilde{\Upsilon}(t, \tau)=\mathcal{I}^{\prime}(t, \tau) \mathcal{P} \tilde{\chi}(t)+\mathcal{K}(\tau) \mathcal{P} \tilde{\Upsilon}(t, \tau)
$$

where the new inhomogeneous term is given by $\mathcal{I}^{\prime}(t, \tau)=$ $\mathcal{I}(\tau) \mathcal{Q} B \mathcal{U}_{0}(t)[\mathbb{1}-\Sigma(t)]^{-1} \Sigma(t) \mathcal{P}$. Eq. (15) is an exact equation of motion for the reduced effective density operator, in which the inhomogeneous term depends on the reduced physical density operator, which obeys the exact equation of motion Eq. (14) with $\mathcal{Q} \tilde{\chi}(0)=0$.

Though Eqs. (15) and (14) are exact, calculating explicit forms for the kernels is difficult. The utility of the projection operator approach used here is that it allows for a systematic expansion in the system-environment coupling strength $\alpha$. Expanding the kernels appearing in Eq. (15) to second order in $\alpha$ and moving back into the Schrödinger picture we find

$$
\partial_{\tau} \Lambda(t, \tau)=i\left[\Lambda(t, \tau), H_{S}\right]+\mathcal{D}(\Lambda(t, \tau))+\mathcal{C}(\varrho(t, \tau)),
$$

where the effective density operator enters through

$$
\mathcal{D}(\Lambda(t, \tau))=-\int_{0}^{\tau} d s \operatorname{Tr}_{E}\left[H_{I},\left[\tilde{H}_{I}(-s), \Lambda(t, \tau) \rho_{R}\right]\right],
$$

and the physical density operator enters through

$$
\mathcal{C}(\varrho(t, \tau))=-\int_{\tau}^{\tau+t} d s \operatorname{Tr}_{E}\left[H_{I}, \tilde{B}(-\tau)\left[\tilde{H}_{I}(-s), \varrho(t, \tau) \rho_{R}\right]\right]
$$

with $\varrho(t, \tau)=U_{S}(\tau) \rho(t) U_{S}^{\dagger}(\tau), \tilde{B}(-\tau)=U_{S}(\tau) B U_{S}^{\dagger}(\tau)$, and we have absorbed factors of $\alpha$ into the interaction Hamiltonians, i.e., $\alpha H_{I} \rightarrow H_{I}$. Let us review what approximations have been made. We assumed factorizing initial conditions, $\chi(0)=\rho(0) \otimes \rho_{R}$, and expanded the kernels to second order in the system-environment coupling strength. From this point onwards no further approximations are necessary. Finally, we note that $\rho(t)$ entering Eq. (18) can be found at no additional cost since to the same level of approximation we have $\partial_{t} \rho(t)=-i\left[H_{S}, \rho(t)\right]+\mathcal{D}(\rho(t))$.

Before proceeding, we note that we can obtain a timeindependent equation of motion for $\Lambda(t, \tau)$ by making a Markovian approximation and let $\tau \rightarrow \infty$ in Eq. (16). We then find $\mathcal{C}(\varrho(t, \tau))=0$ and the inhomogeneous term disappears. In this case the regression theorem is recovered since $\rho(t)$ and $\Lambda(t, \tau)$ obey the same equation of motion. Recalling that we also find $\mathcal{C}(\varrho(t, \tau))=0$ when making the Born approximation, $\chi(t) \approx \rho(t) \otimes \rho_{R}$, we conclude that in the present context the Markovian approximation cannot be made without also implicitly making the Born approximation. Is the converse also true? Is it possible to not make the Markovian approximation by leaving the integration limit in Eq. (16) at $\tau$, yet at the same time make the Born approximation and neglect the inhomogeneous term $\mathcal{C}(\varrho(t, \tau))$ ? This is what one would obtain naively applying the regression theorem to a non-Markovian master equation for the physical density operator. In the following we will see that this approach is ill advised and can give rise to unphysical results. 


\section{APPLICATION TO A DRIVEN SEMICONDUCTOR QUANTUM DOT COUPLED TO ACOUSTIC PHONONS}

We now use our results and consider a driven semiconductor QD in a non-Markovian acoustic phonon environment $[14,24,27]$. The QD is described by ground and single exciton states $|g\rangle$ and $|e\rangle$, and the laser by a constant Rabi frequency $\Omega$ and detuning $\delta$. In a rotating frame, and within the dipole and rotating wave approximations the Hamiltonian is given by $H=H_{S}+H_{I}+H_{E}$, with $H_{S}=\delta \sigma^{\dagger} \sigma+(\Omega / 2)\left(\sigma^{\dagger}+\sigma\right), \quad H_{I}=\sigma^{\dagger} \sigma \sum_{k} g_{k}\left(b_{k}^{\dagger}+\right.$ $b_{k}$ ), and $H_{E}=\sum_{k} \omega_{k} b_{k}^{\dagger} b_{k}$, where $\sigma=|g\rangle\langle e|$, a phonon with wave vector $k$ and frequency $\omega_{k}$ is described by creation and annihilation operators $b_{k}^{\dagger}$ and $b_{k}$, and we take a thermal state for the phonon environment $\rho_{R}=$ $\exp \left[-H_{E} / k_{B} T\right] / \operatorname{Tr}\left[\exp \left(-H_{E} / k_{B} T\right)\right]$, with $T$ being the sample temperature. The exciton-phonon interaction is characterized by coupling constants $g_{k}$, which ultimately enter only through the spectral density $J(\omega)=\sum_{k} g_{k}^{2} \delta\left(\omega-\omega_{k}\right)$. For coupling to longitudinal acoustic phonons we can take the form $J(\omega)=\eta \omega^{3} \exp \left[-\left(\omega / \omega_{c}\right)^{2}\right]$, with $\eta$ being the QD-phonon coupling strength and $\omega_{c}$ being the cutoff frequency, whose inverse gives the memory time of the environment [27]. We tune the laser to the phonon-shifted QD transition frequency, $\delta=\int_{0}^{\infty} d \omega J(\omega) / \omega$, set $\Omega=0.12 \mathrm{ps}^{-1}$, and use the realistic parameters $\eta=0.03 \mathrm{ps}^{2}$ and $\omega_{c}=2.2 \mathrm{ps}^{-1}$, with $T=4 \mathrm{~K}$. The steady-state first-order correlation function of the QD emission is $g^{(1)}(\tau)=\lim _{t \rightarrow \infty}\left\langle\sigma^{\dagger}(t+\tau) \sigma(t)\right\rangle$, which we calculate with Eq. (16), adding a term $\Gamma\left[\sigma \Lambda(t, \tau) \sigma^{\dagger}-\right.$ $\left.\frac{1}{2}\left\{\sigma^{\dagger} \sigma, \Lambda(t, \tau)\right\}\right]$ with $1 / \Gamma=100$ ps to capture spontaneous emission. Including spontaneous emission in this way assumes that the photonic environment is strictly Markovian and is justified fully in the Appendix. Having obtained the firstorder correlation function the incoherent emission spectrum is defined as $S(\Delta \omega)=\operatorname{Re}\left\{\int_{0}^{\infty} d \tau\left[g^{(1)}(\tau)-g^{(1)}(\infty)\right] \mathrm{e}^{-i \Delta \omega \tau}\right\}$.

Figure 1 shows the real (a) and imaginary (b) parts of $g^{(1)}(\tau)$ calculated using the Markovian approximation [taking $\tau \rightarrow \infty$ in Eqs. (17) and (18), solid blue], the full non-Markovian theory (dashed orange), and using the naive non-Markovian theory (neglecting the inhomogeneous term in Eq. (18), dotted green). We see that for times less than the environment correlation time of $\sim 1 \mathrm{ps,} \mathrm{all} \mathrm{three} \mathrm{theories}$ predict quite distinct behavior, reflecting the fact that nonMarkovian effects are most important and these time scales. Figure 1(c) shows the corresponding incoherent emission spectrum, which on the inset scale displays the well-known Mollow triplet. From the main part of Fig. 1(c), we see that the Markovian theory, which predicts no short time oscillations, correspondingly predicts no spectral features at large frequencies. The full non-Markovian theory, however, predicts a broad sideband at lower emission frequencies. This sideband is well known experimentally [20,43-45] and is attributed to phonon emission, which our theory supports. Thus, the phonon sideband in the emission spectrum is a signature of non-Markovian behavior. This is a key feature of this work; observation of non-Markovian behavior of one-time expectation values typically necessitates initializing a system in a well-defined state and tracking dynamics on very short time scales (ps in this example). Steady-state two-time correlation functions, on the other hand, capture fluctuations of
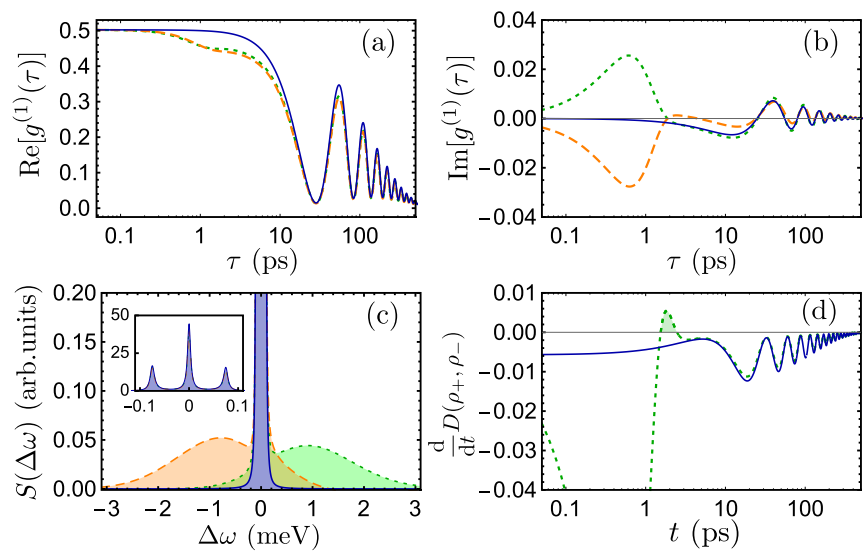

FIG. 1. Real (a) and imaginary (b) parts of the first-order correlation function, calculated using a Markovian approximation (solid blue), the full non-Markovian theory (dashed orange), and the non-Markovian theory but neglecting the inhomogeneous term in Eq. (18) (green dotted). Plot (c) shows the corresponding emission spectrum with the inset showing a different scale on which the Mollow triplet can be seen. From the main part of plot (c) it is seen that only the full non-Markovian theory correctly captures the phonon sideband at lower energies. Plot (d) shows the derivative of the trace distance between two states evolved from different initial conditions, whose positive values for times $\sim 1$ ps demonstrates backflow of information and true non-Markovianity.

a system from equilibrium. Non-Markovian behavior of these fluctuations can be much more readily observed since their Fourier transform corresponds to an emission spectrum [46].

We note that while the phonon sideband has been calculated previously, it has so only in the zero driving limit $\Omega \rightarrow 0$ where the model becomes exactly solvable and the Mollow triplet is not present [43-45,47]. The theory presented here works for nonzero $\Omega$, allowing us to calculate the fraction of power emitted into the phonon sideband, which for the realistic parameters used here gives $\sim 10 \%$, in good agreement with recent experiments [20].

Interestingly, it can be seen that the naive non-Markovian theory predicts a sideband at higher energies, in contrast to both intuition and experimental evidence. The inhomogeneous term in Eq. (18), which the naive approach ignores, captures deviations of the true state of the environment from the reference state $\rho_{R}$ used in the master equation. For the emission spectrum, these deviations are important, since we assumed $\rho_{R}$ to be a thermal state with respect to the QD ground state, which is not the correct initial condition for an emission process. This reveals why in neglecting the inhomogeneous term the sideband incorrectly appears at higher energies; since it assumes the environment to be in equilibrium with respect to the QD ground state, it inadvertently gives dynamics which correspond more to an absorption spectrum. We note that this correspondence is only approximate and is not expected to be a general feature.

The steady-state correlation function we have calculated captures fluctuations of the QD about its steady state, and our results suggest these fluctuations are non-Markovian in nature. In order for this is be confirmed, we calculate a non-Markovianity witness in the form of the derivative of the trace distance $D\left(\rho_{+}, \rho_{-}\right)=\frac{1}{2}\left|\rho_{+}(t)-\rho_{-}(t)\right|$, where 
$\rho_{+}(t)$ and $\rho_{-}(t)$ are physical density operator states evolved from two different initial states $\rho_{ \pm}(0)=\frac{1}{2}\left(\mathbb{1} \pm \sigma_{y}\right)$ with $\sigma_{y}=-i|e\rangle\langle g|+i| g\rangle\langle e|[36]$. We are interested here in the evolution of reduced physical density operators since these characterize the behavior of physical QD exciton, and as such use the equation of motion $\partial_{t} \rho(t)=-i\left[H_{S}, \rho(t)\right]+\mathcal{D}(\rho(t))$ (i.e., without inhomogeneous term). A positive derivative of the trace distance is interpreted as a flow of information from the environment into the system and is a sufficient condition to prove indivisibility of the underlying dynamical map, both of which can be considered definitions of nonMarkovianity $[10,36,48]$. In Fig. $1(\mathrm{~d})$ we show $\frac{d}{d t} D\left(\rho_{+}, \rho_{-}\right)$ calculated using the non-Markovian theory (dotted, green), and within the Markovian approximation (solid, blue). We see that our non-Markovian theory gives rise to a time interval during which the derivative is positive, confirming true non-Markovian behavior.

\section{SUMMARY}

We have developed an extension to the quantum regression theorem, valid to second order in the system-environment coupling strength, and invoking the Born approximation at a single fixed initial time. These results have been used to demonstrate that phonon sidebands in the resonance fluorescence emission spectra of a QD are a signature of non-Markovian behavior. In this context, it was shown that this non-Markovian behavior is associated with a flow of information from the phonon environment back into the QD exictonic system, which is a sufficient condition to prove indivisibility of the underlying dynamical map. The projection operator method used here is an ideal starting point to include higher order system-environment coupling terms, which can in some cases lead to an exact resummation [30]. Finally, it will be interesting to investigate how the results obtained here can be used to optically quantity non-Markovian behavior [10,36,48-50].

\section{ACKNOWLEDGMENTS}

I would like to thank Jesper Mørk, Ahsan Nazir and Jake Iles-Smith for useful discussions. This work was funded by project SIQUTE (Contract No. EXL02) of the European Metrology Research Programme (EMRP). The EMRP is jointly funded by the EMRP participating countries within EURAMET and the European Union.

\section{APPENDIX A: EFFECTIVE DENSITY OPERATOR MASTER EQUATION FOR TIME-DEPENDENT INTERACTION HAMILTONIANS}

Here we give an extension to the results provided in the main text which facilitates the inclusion of time-dependent interaction Hamiltonians. For a time-dependent interaction Hamiltonian we can write the complete Schrödiner picture Hamiltonian in the form $H(t)=H_{S}+\alpha H_{I}(t)+H_{E}$. In this case defining an interaction picture proceeds analogously as in the main text, and the interaction picture equation of motion for the effective density operator again takes the form of Eq. (3), though now we have

$$
\tilde{H}_{I}(\tau)=U_{0}^{\dagger}(\tau) H_{I}(\tau) U_{0}(\tau)
$$

with $H_{I}(\tau)$ the Schrödinger picture interaction Hamiltonian at time $\tau$, and $\tilde{\Upsilon}(t, \tau)=U_{0}^{\dagger}(\tau) U(t+\tau, t)[B \chi(t)] U^{\dagger}(t+$ $\tau, t) U_{0}(\tau)$ where the time evolution operator satisfies $i \partial_{t} U\left(t, t_{0}\right)=H(t) U\left(t, t_{0}\right) \quad$ with $U\left(t_{0}, t_{0}\right)=\mathbb{1}$. For this time-dependent interaction Hamiltonian the derivation of the effective density operator master equation proceeds precisely as in the main text, and we again arrive at the general expression in Eq. (15), the only difference being that the implicit occurrences of the interaction Hamiltonians are defined through Eq. (A1). Expanding to second order in the system-environment coupling strength proceeds analogously, though some care must be taken when moving back into the Schrödinger picture. For a time-dependent Hamiltonian the Schrödinger picture equation of motion for the effective density again has the form $\partial_{\tau} \Lambda(t, \tau)=$ $-i\left[H_{S}, \Lambda(t, \tau)\right]+\mathcal{D}(\Lambda(t, \tau))+\mathcal{C}(\varrho(t, \tau))$, though now

$$
\begin{aligned}
\mathcal{D}(\Lambda(t, \tau))= & -\int_{0}^{\tau} d s \operatorname{Tr}_{E}\left[\tilde{H}_{I}(\tau, 0),\right. \\
& \left.\times\left[\tilde{H}_{I}(\tau-s,-s), \Lambda(t, \tau) \rho_{R}\right]\right],
\end{aligned}
$$

and the inhomogeneous term is given by

$$
\begin{aligned}
\mathcal{C}(\rho(t))= & -\int_{\tau}^{\tau+t} d s \operatorname{Tr}_{E}\left[\tilde{H}_{I}(\tau, 0), \tilde{B}(-\tau)\right. \\
& \left.\times\left[\tilde{H}_{I}(t+\tau-s,-s), \varrho(t, \tau) \rho_{R}\right]\right],
\end{aligned}
$$

and we have defined $\tilde{H}_{I}\left(t_{1}, t_{2}\right)=U_{0}^{\dagger}\left(t_{2}\right) H_{I}\left(t_{1}\right) U_{0}\left(t_{2}\right)$. Note that in order to recover the case for a time-independent interaction Hamiltonian we simply set the first time argument in $\tilde{H}_{I}\left(t_{1}, t_{2}\right)$ to zero.

\section{APPENDIX B: INCLUSION OF SPONTANEOUS EMISSION WITHIN THE MARKOVIAN APPROXIMATION}

Here we give details of how spontaneous emission can be included into the effective density operator master equation in the context of the quantum dot (QD) example in the main text. To so so we consider an optically driven QD coupled to both a phonon and photon reservoir. Within the dipole and rotating wave approximations the total Schrödinger picture Hamiltonian in a frame rotating at the laser frequency $\omega_{l}$ takes the form $H(t)=H_{S}+H_{I 1}+H_{I 2}(t)+H_{E 1}+H_{E 2}$, where $H_{S}=\delta \sigma^{\dagger} \sigma+(\Omega / 2)\left(\sigma^{\dagger}+\sigma\right), H_{I 1}=\sigma^{\dagger} \sigma \sum_{k} g_{k}\left(b_{k}^{\dagger}+\right.$ $\left.b_{k}\right), H_{E 1}=\sum_{k} \omega_{k} b_{k}^{\dagger} b_{k}$, while

$$
H_{I 2}(t)=\sum_{q} h_{q}\left(\sigma a_{q}^{\dagger} e^{-i \omega_{l} t}+\sigma^{\dagger} a_{q} e^{i \omega_{l} t}\right),
$$

and $H_{E 2}=\sum_{q} v_{q} a_{q}^{\dagger} a_{q}$, where parameters with a $k$ subscript refer to phonons, while $h_{q}$ is the coupling constant between the quantum dot and photonic mode $q$, described by creation operator $a_{q}^{\dagger}$ and frequency $v_{q}$. Since the total interaction Hamiltonian $H_{I}(t)=H_{I 1}+H_{I 2}(t)$ is time dependent we must use Eqs. (A2) and (A3), where the trace is now taken over both phonon and photon degrees of freedom. Assuming that $H_{I 1}$ and $H_{I 2}(t)$ contain no environment operators that act in the same Hilbert space (as is the case in our example), one finds that provided $\operatorname{Tr}_{E 1}\left[H_{I 1} \rho_{R}\right]=0$, the cross terms mixing $H_{I 1}$ and $H_{I 2}(t)$ in Eqs. (A2) and (A3) vanish, and we can write $\partial_{\tau} \Lambda(t, \tau)=-i\left[H_{S}, \Lambda(t, \tau)\right]+\mathcal{D}_{1}(\Lambda(t, \tau))+$ $\mathcal{C}_{1}(\varrho(t, \tau))+\mathcal{D}_{2}(\Lambda(t, \tau))+\mathcal{C}_{2}(\varrho(t, \tau))$, where $\mathcal{D}_{1}$ and $\mathcal{C}_{1}$ 
contain only phonon terms, i.e., they are Eqs. (A2) and (A3) with $\tilde{H}_{I}\left(t_{1}, t_{2}\right) \rightarrow U_{0}^{\dagger}\left(t_{2}\right) H_{I 1} U_{0}\left(t_{2}\right)$, and $\mathcal{D}_{2}$ and $\mathcal{C}_{2}$ contain only photon terms, i.e., Eqs. (A2) and (A3) with $\tilde{H}_{I}\left(t_{1}, t_{2}\right) \rightarrow$ $U_{0}^{\dagger}\left(t_{2}\right) H_{I 2}\left(t_{1}\right) U_{0}\left(t_{2}\right)$. As in the main text we have $U_{0}(t)=$ $U_{S}(t) U_{E}(t)$ though now $U_{E}(t)=\exp \left[-i\left(H_{E 1}+H_{E 2}\right) t\right]$.

Let us consider the term in $\mathcal{D}_{2}(\Lambda(t, \tau))$ in more detail. The relevant interaction Hamiltonian can be written

$$
\tilde{H}_{I 2}(\tau-s,-s)=\tilde{\sigma}(-s) \tilde{A}^{\dagger}(-s) e^{-i \omega_{l}(\tau-s)}+\text { H.c. },
$$

where $\tilde{\sigma}(-s)=e^{-i H_{S} s} \sigma e^{i H_{S} s}$ and $\tilde{A}(-s)=\sum_{q} h_{q} a_{q} e^{i v_{q} s}$. Assuming a zero-temperature thermal state environment for the photons, i.e., $\rho_{R}=\rho_{R 1} \rho_{R 2}$ with $\rho_{R 1}$ the state of the phonon environment and $\rho_{R 2}=$ $\exp \left[-\beta \sum_{q} v_{q} a_{q}^{\dagger} a_{q}\right] / \operatorname{Tr}\left[\exp \left(-\beta \sum_{q} v_{q} a_{q}^{\dagger} a_{q}\right)\right] \quad$ with $\beta \rightarrow \infty$, we find $\operatorname{Tr}_{E}\left[A^{\dagger} \tilde{A}^{\dagger}(-s) \rho_{R}\right]=\operatorname{Tr}_{E}\left[A \tilde{A}(-s) \rho_{R}\right]=$ $\operatorname{Tr}_{E}\left[A^{\dagger} \tilde{A}(-s) \rho_{R}\right]=0$, and we are left with

$$
\begin{aligned}
\mathcal{D}_{2}(\Lambda(t, \tau))= & -\int_{0}^{\tau} d s \operatorname{Tr}_{E}\left[A \tilde{A}^{\dagger}(-s) \rho_{R}\right] e^{i \omega_{l} s} \\
& \times\left(\sigma^{\dagger} \tilde{\sigma}(-s) \Lambda(t, \tau)-\tilde{\sigma}(-s) \Lambda(t, \tau) \sigma^{\dagger}\right) \\
& + \text { H.c. }
\end{aligned}
$$

We now make a Markovian approximation, with respect to the photon environment only, and approximate the remaining correlation function as a $\delta$ function; i.e., we take $\operatorname{Tr}_{E}\left[A \tilde{A}^{\dagger}(-s) \rho_{R}\right]=\zeta \delta(s)$, in which case we find

$$
\mathcal{D}_{2}(\Lambda(t, \tau))=\Gamma\left(\sigma \Lambda(t, \tau) \sigma^{\dagger}-\frac{1}{2}\left\{\sigma^{\dagger} \sigma, \Lambda(t, \tau)\right\}\right),
$$

where $\Gamma=2 \zeta$ is the spontaneous emission rate. Considering now the photonic inhomogeneous term, $\mathcal{C}_{2}(\varrho(t, \tau))$, making the same Markovian approximation for a zerotemperature environment results in $\mathcal{C}_{2}(\varrho(t, \tau))=0$ for all times $\tau>0$ of interest owing to the integration limits in Eq. (A3). As such, within the Markovian approximation for the photonic environment, we can simply neglect the photon terms at a Hamiltonian level, provided we add a term equal to Eq. (B4) to the equation of motion Eq. (16) in the main text. We note that approximating the photonic correlation functions as $\delta$ functions is expected to be a good approximation for quantum dots in free space or in low $Q$-factor cavities, where photon correlation times of $\sim 10^{-2}$ to $10^{-3}$ ps are typically orders of magnitude shorter than the phonon bath correlation time of $\sim 1$ ps [24,26].
[1] B. R. Mollow, Phys. Rev. 188, 1969 (1969).

[2] H. J. Carmichael, Statistical Methods in Quantum Optics (Springer, New York, 1998).

[3] S. Swain, J. Phys. A 14, 2577 (1999).

[4] D. Alonso and I. de Vega, Phys. Rev. Lett. 94, 200403 (2005).

[5] I. de Vega and D. Alonso, Phys. Rev. A 77, 043836 (2008).

[6] C. Flindt, T. Novotný, A. Braggio, M. Sassetti, and A. P. Jauho, Phys. Rev. Lett. 100, 150601 (2008).

[7] A. Budini, J. Stat. Phys. 131, 51 (2008).

[8] H.-S. Goan, C.-C. Jian, and P.-W. Chen, Phys. Rev. A 82, 012111 (2010).

[9] H.-S. Goan, P.-W. Chen, and C.-C. Jian, J. Chem. Phys. 134, 124112 (2011).

[10] G. Guarnieri, A. Smirne, and B. Vacchini, Phys. Rev. A 90, 022110 (2014).

[11] L. Mandel and E. Wolf, Optical Coherence and Quantum Optics (Cambridge University Press, Cambridge, UK, 1995).

[12] K. Koshino and A. Shimizu, Phys. Rev. Lett. 92, 030401 (2004).

[13] E. B. Flagg, A. Muller, J. W. Robertson, S. Founta, D. G. Deppe, M. Xiao, W. Ma, G. J. Salamo, and C. K. Shih, Nat. Phys. 10, 1038 (2009).

[14] A. J. Ramsay, A. V. Gopal, E. M. Gauger, A. Nazir, B. W. Lovett, A. M. Fox, and M. S. Skolnick, Phys. Rev. Lett. 104, 017402 (2010).

[15] L. Monniello, C. Tonin, R. Hostein, A. Lemaitre, A. Martinez, V. Voliotis, and R. Grousson, Phys. Rev. Lett. 111, 026403 (2013).

[16] S. Ates, S. M. Ulrich, S. Reitzenstein, A. Loffler, A. Forchel, and P. Michler, Phys. Rev. Lett. 103, 167402 (2009).

[17] S. M. Ulrich, S. Ates, S. Reitzenstein, A. Loffler, A. Forchel, and P. Michler, Phys. Rev. Lett. 106, 247402 (2011).

[18] Y.-J. Wei, Y. He, Y. M. He, C. Y. Lu, J. W. Pan, C. Schneider, M. Kamp, S. Hofling, D. P. S. McCutcheon, and A. Nazir, Phys. Rev. Lett. 113, 097401 (2014).
[19] C. Matthiesen, A. N. Vamivakas, and M. Atatüre, Phys. Rev. Lett. 108, 093602 (2012).

[20] C. Matthiesen, M. Geller, C. H. H. Schulte, C. Le Gall, J. Hansom, Z. Li, M. Hugues, E. Clarke, and M. Atatüre, Nat. Commun. 4, 1600 (2013).

[21] P. Michler et al., Science 290, 2282 (2000).

[22] C. Santori et al., Nature (London) 419, 594 (2002).

[23] E. B. Flagg, A. Muller, S. V. Polyakov, A. Ling, A. Migdall, and G. S. Solomon, Phys. Rev. Lett. 104, 137401 (2010).

[24] D. P. S. McCutcheon and A. Nazir, Phys. Rev. Lett. 110, 217401 (2013).

[25] C. Roy and S. Hughes, Phys. Rev. Lett. 106, 247403 (2011).

[26] K. Roy-Choudhury and S. Hughes, Opt. Lett. 40, 1838 (2015).

[27] D. P. S. McCutcheon and A. Nazir, New J. Phys. 12, 113042 (2010).

[28] P. Kaer and J. Mørk, Phys. Rev. B 90, 035312 (2014).

[29] Ł. Cywiński, W. M. Witzel, and S. Das Sarma, Phys. Rev. B 79, 245314 (2009).

[30] E. Barnes, Ł. Cywiński, and S. Das Sarma, Phys. Rev. Lett. 109, 140403 (2012).

[31] N. Ubbelohde, K. Roszak, F. Hohls, N. Maire, R. J. Haug, and T. Novotný, Sci. Rep. 2, 374 (2012).

[32] N. H. Lindner and T. Rudolph, Phys. Rev. Lett. 103, 113602 (2009).

[33] O. Gazzano et al., Nat. Commun. 4, 1425 (2013).

[34] M. Müller, S. Bounouar, K. D. Jöns, M. Glässl, and P. Michler, Nat. Photon. 8, 224 (2014).

[35] D. P. S. McCutcheon, N. H. Lindner, and T. Rudolph, Phys. Rev. Lett. 113, 260503 (2014).

[36] H.-P. Breuer, E.-M. Laine, and J. Piilo, Phys. Rev. Lett. 103, 210401 (2009).

[37] H.-P. Breuer and F. Petruccione, The Theory of Open Quantum Systems (Oxford University Press, Oxford, UK, 2002). 
[38] S. Nakajima, Prog. Theor. Phys. 20, 948 (1958).

[39] R. Zwanzig, J. Chem. Phys. 33, 1338 (1960).

[40] F. Shibata, Y. Takahashi, and N. Nashitsume, J. Stat. Phys. 17, 171 (1977).

[41] D. P. S. McCutcheon, N. S. Dattani, E. M. Gauger, B. W. Lovett, and A. Nazir, Phys. Rev. B 84, 081305(R) (2011).

[42] S. Jang, J. Chem. Phys. 131, 164101 (2009).

[43] L. Besombes, K. Kheng, L. Marsal, and H. Mariette, Phys. Rev. B 63, 155307 (2001).

[44] I. Favero, G. Cassabois, R. Ferreira, D. Darson, C. Voisin, J. Tignon, C. Delalande, G. Bastard, P. Roussignol, and J. M. Gérard, Phys. Rev. B 68, 233301 (2003).

[45] K. J. Ahn, J. Förstner, and A. Knorr, Phys. Rev. B 71, 153309 (2005).
[46] We note that numerical investigations reveal that for certain parameters the non-Markovian theory can predict spectra which take on slightly negative values. It is believed that this signifies a limitation of the second-order approximation used, though it may also point towards the need for a refined definition of the commonly used steady-state emission spectrum.

[47] K. Roy-Choudhury and S. Hughes, Phys. Rev. B 92, 205406 (2015).

[48] Á. Rivas, S. F. Huelga, and M. B. Plenio, Phys. Rev. Lett. 105, 050403 (2010).

[49] M. M. Wolf, J. Eisert, T. S. Cubitt, and J. I. Cirac, Phys. Rev. Lett. 101, 150402 (2008).

[50] K. Luoma, P. Haikka, and J. Piilo, Phys. Rev. A 90, 054101 (2014). 